\title{
177. A Study of the Chromosomes and Enzymatic Patterns of Sticklebacks of Japan*)
}

\author{
By Jun-ichi Muramoto,**) Kiyoshi Igarashi,***) \\ Masahiro ITOH,**) and Sajiro MAKINo**) \\ (Comm. by Yoshimaro Tanaka, M. J. A., Nov. 12, 1969)
}

On conventional ecological and morphological bases, classification of the family Gasterosteidae has been in controversy, because of many confusing body characters.

The previous study by Muramoto and Igarashi (1969) presented some chromosomal and enzymatic features critical for classification of Gasterosteus aculeatus aculeatus, G. a. microcephalus and Pungitius sinensis. In the hope of collecting additional data useful as criteria for the classification and evolution for sticklebacks, an investigation of the chromosomes and enzymatic patterns of glucose-6-phosphate dehydrogenase (G-6-PD) and galactose-6-phosphate dehydrogenase (Gal-6-PD) was undertaken.

Materials and methods. The sticklebacks studied here are: The anadromous and the land-locked type of Gasterosteus aculeatus aculeatus (L.), G. a. microcephalus (L.), the standard and the trachura type of Pungitius pungitius (L.), P. tymensis (Nikolsky), P. sinensis (Guichenot) and Pungitius sp. Spleen, gill and/or kidney from each species supplied materials for chromosome study. Prior to sacrifice, each individual received intramuscular injection of 0.1 to $0.2 \mathrm{ml}$ colchicine solution for 2.0 to 2.5 hours. Upon sacrifice, small pieces of tissue were put into $0.075 \mathrm{M} \mathrm{KCl}$ for 20 minutes, and then fixed with Carnoy's solution. After 1 hour of fixation, cells on slides were flamed and stained with Giemsa solution. For enzymatic studies, skeletal muscles were frozen on dry ice immediately after removal, homogenized with 3 volumes of homogenizing solution and centrifuged at $15,000 \mathrm{~g}$ for 20 minutes. After electrophoresis for about 2 hours at room temperature or in the refrigerator, the gels were cut in half longitudinally; one half was stained with a solution containing glucose-6-phosphate as a substrate, and the other half was stained with a solution containing galactose-6-phosphate as a substrate at $37^{\circ} \mathrm{C}$.

*) Contributions from the Chromosome Research Unit, Faculty of Science, Hokkaido University, Sapporo.

**) Chromosome Research Unit, Hokkaido University.

***) Fukui Educational Center, Fukui. 
Findings. Gasterosteus aculeatus aculeatus (L.). The chromosomes showed no visible variation between the anadromous and the land-locked type of this species. The diploid complement consisted of 42 chromosomes which were arranged into 8 pairs of meta- and submetacentric chromosomes, 5 pairs of subtelocentrics and 8 pairs of acrocentrics (Fig. 1). However, a slight difference between them was detected in the enzymatic pattern of G-6-PD: the anadromous type showed two more bands, $F$ and $G$, in the anodal direction than the land-locked type (Fig. 5). The Gal-6-PD pattern of the anadromous type was characterized by $\mathrm{A}, \mathrm{B}$ and $\mathrm{D}$ bands, in contrast to the Gal-6-PD pattern of the land-locked type which showed A and B bands (Fig. 5).

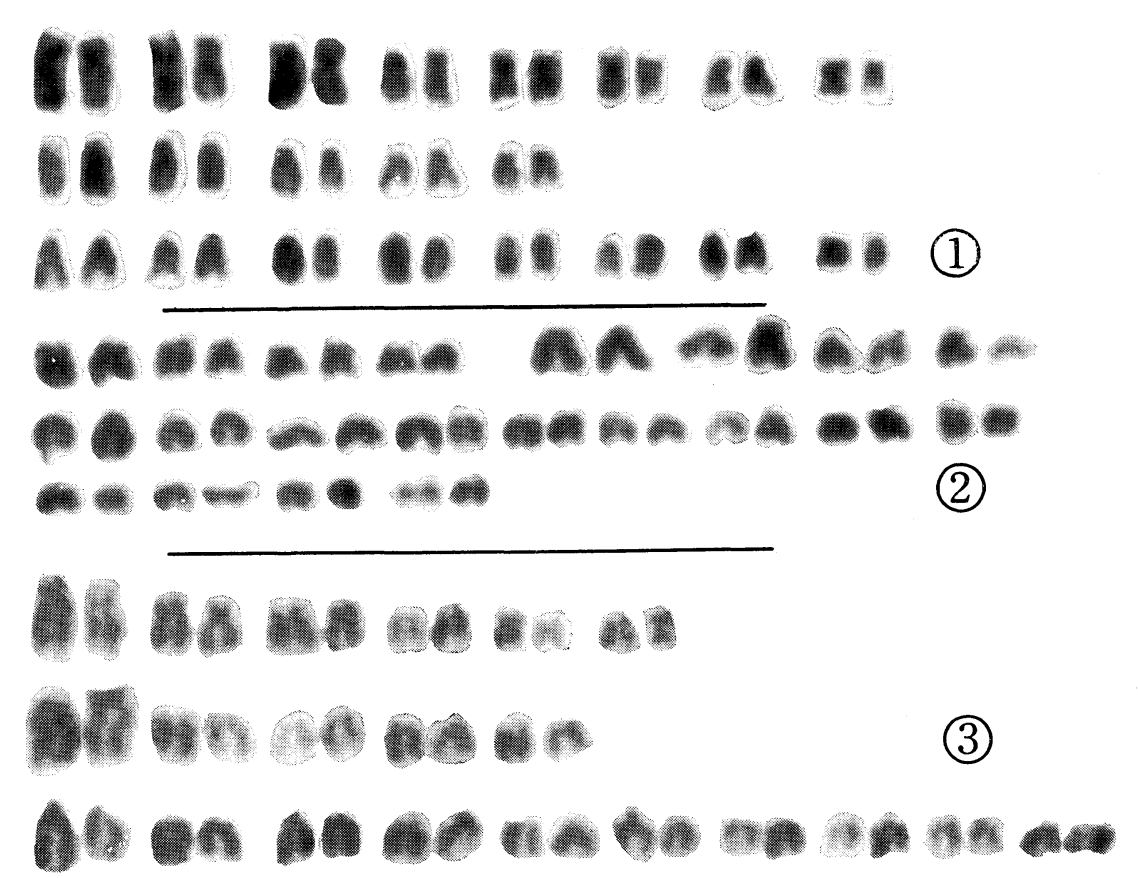

Fig. 1. Karyotype of the anadromous type of Gasterosteus a. aculeatus (L.) from the gill: 8 pairs of meta- and submetacentrics on top row, 5 pairs of subterminals on middle row, and 8 pairs of acrocentrics on bottom row.

Fig. 2. Karyotype from the gill of Pungitius sinensis (Guichenot): 4 pairs of meta- and submetacentrics and 4 subterminals on the first row, 13 pairs of acrocentrics on the second and third rows.

Fig. 3. Karyotype of Pungitius sp. from the gill: 6 pairs of meta- and submetacentrics on top row, 5 pairs of subterminals and 10 pairs of acrocentrics on middle and bottom rows.

Gasterosteus a. microcephalus (L.). This species had a karyotype morphologically identical with that of the anadromous and the land-locked type of Gasterosteus a. aculeatus (L.). Also, no partic- 
ular difference appeared in the zymogram of G-6-PD between the present species and the land-locked type of G. a. aculeatus (L.) (Figs. 4 and 5).

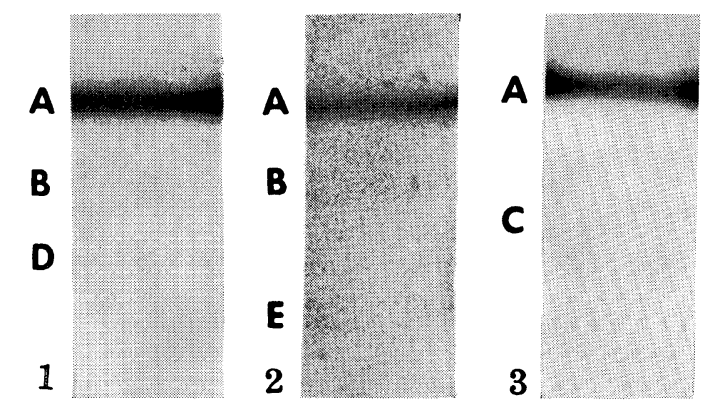

Fig. 4. Electrophoretic patterns of G-6-PD. 1) land-locked type of Gasterosteus a. aculeatus (L.); 2) P. sinensis (Guichenot), and 3) P. tymensis (Nikolsky).

Pungitius pungitius (L.). The chromosome number of this species was the same as that of $G$. a. aculeatus, 2 n being 42 in both the standard and the trachura type. The karyotype was slightly different: there were 6 pairs of meta- and submetacentrics, 5 pairs of subtelocentrics and 10 pairs of acrocentrics. The submetacentrics forming the 1st pair were larger than the other submetacentrics or metacentrics showing a gradual seriation in size. The 1st subtelocentric pair was the largest element of all. The zymograms of this species showed A and C bands for G-6-PD as well as for Gal-6-PD, both the standard and trachura types being identical (Fig. 5). The activity of the $\mathrm{C}$ band was observed to be stronger than that of the A band.
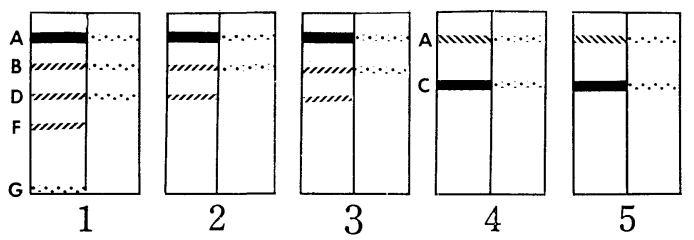

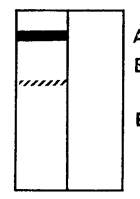

6

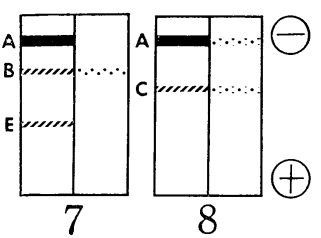

Fig. 5. Diagrams of G-6-PD and Gal-6-PD patterns from skeletal muscle. 1. Anadromous type of Gasterosteus a. aculeatus (L.). 2. Land-locked type of Gasteroteus a. aculeatus (L.). 3. Gasterosteus a. microcephalus (L.). 4. Standard type of Pungitius pungitius (L.). 5. Trachura type of $P$. pungitius (L.). 6. P. tymensis (Nikolsky). 7. P. sinensis (Guichenot). 8. Pungitius sp. Black, oblique and dotted lines show the strong, middle and weak activity, respectively.

Pungitius tymensis (Nikolsky). No identifiable difference in the chromosome constitution was detected between the present species 
and $P$. pungitius. In the enzymatic pattern of G-6-PD, the activity of the A band was stronger than that of the $\mathrm{C}$ band (Figs. 4 and 5). However, the Gal-6-PD pattern of this species did not show any active band.

Pungitius sinensis (Guichenot). A slight difference was detected in the karyotype between this species and other species of Pungitius. The diploid complement of this species is composed of 4 pairs of submeta- and metacentrics, 4 pairs of subtelocentrics and 13 pairs of acrocentrics, in contrast to that of other species which was composed of 6 pairs of submeta- and metacentrics, 5 pairs of subtelocentrics, and 10 pairs of acrocentrics. The pattern of G-6-PD showed stronger activity in the $\mathrm{A}$ band than in that of other $\mathrm{B}$ and $\mathrm{E}$ bands. The $\mathrm{B}$ band was detected in the pattern of Gal-6-PD (Figs. 4 and 5).

Pungitius sp. The karyotype of this species was apparently the same as that of the Pungitius species mentioned above (Fig. 3). This species was also identical with $P$. tymensis (Nikolsky) in the pattern of G-6-PD. The standard and the trachura type of $P$. pungitius (L.) showed a similar pattern of Gal-6-PD (Fig. 5).

Discussion. It has been shown that the production of glucose6-phosphate dehydrogenase (G-6-PD) is related to a sex-linked gene in man and some other mammals (Childs et al. 1958, Young et al. 1964, Ohno et al. 1965, Trujillo et al. 1965). The preliminary study (Muramoto and Igarashi 1969) gave no evidence for the above enzyme-gene relationship. The present study revealed that the enzymatic pattern of G-6-PD displayed no sexual differences in the standard type of Pungitius pungitius. For critical statement on the above findings, further investigation is needed with extensive material.

In this study the chromosome constitution and the enzymatic patterns of G-6-PD and Gal-6-PD of the land-locked and the ariadromous type of Gasterosteus aculeatus aculeatus were compared. So far as the scope of the present investigation is concerned, the enzymatic pattern of the anadromous type differed from those of the landlocked type. From these findings, it may be assumed that environmental factor (s) might induce in some way metabolic changes in the living system.

Ikeda (1950) suggested that the hybrid between $P$. pungitius and $P$. sinensis might not be produced. The difference of the chromosome constitution and the enzymatic pattern occurring between the two species seems to support the above view.

The present study has demonstrated a striking similarity of the zymograms and the chromosome constitutions between the standard and the trachura type of $P$. pungitius, and also between $P$. tymensis 
and Pungitius sp. Evidence presented seems to be useful as criteria for the systematic evaluation of closely related forms or species of the sticklebacks.

The literature indicates that the chromosome number of 42 has been reported in $P$. pungitius and $P$. tymensis by Makino (1934) with the old testis-section method.

Summary. The chromosomes and the enzymatic patterns of glucose-6-phosphate dehydrogenase and galactose-6-phosphate dehydrogenase were investigated in the anadromous and the land-locked type of Gasterosteus a aculeatus (L.), G. a. microcephalus (L.), the standard and the trachura type of Pungitius pungitius (L.), Pungitius tymensis (Nikolsky), Pungitius sinensis (Guichenot) and Pungitius sp. The data obtained seem to be significant as criteria for taxonomical evaluation in diagnosing closely related forms or allied species of the sticklebacks.

Acknowledgement. We are grateful to Dr. Kenkichi Ishigaki for supplying P. tymensis and also to Miss Setsuko Takahashi for her friendly assistance in collecting $P$. pungitius and microscopic observations.

\section{References}

Childs, B., W. Zinkham, E. A. Browne, E. L. Kimbro, and J. V. Torbert (1958): Bull. John Hopkins Hosp., 102, 21-37.

Ikeda, K. (1950): Oguma commemoration volume on cytology and genetics, Part 2, pp. 29-37.

Makino, S. (1934): Cytologia, 5, 155-168.

Muramoto, J., and K. Igarashi (1969): Jour. Fac. Sci. Hokkaido Uuiv. Ser. VI, Zoology, 17, 266-270.

Ohno, S., J. Pool, and I. Gustavson (1965): Science, 150, 1737-1738.

Trujillo, J. M., B. Walden, P. O'Neil, and H. B. Anstall (1965): Science, 148, 1603-1604.

Young, W. J., J. E. Porter, and B. Childs (1964): Science, 143, 140-141. 